\title{
Étude du régime alimentaire de deux souches de truite arc-en-ciel marocaine et bulgare dans deux plans d'eau du Moyen-Atlas Amghass I et Amghass II Dietary regime of moroccan and bulgarian rainbow trout in two locations of the Moyen-Atlas (Amghass I and Amghass II) in Morocco
}

\author{
L. Ibn Majdoub Hassani, M. Khodari et M. Alaoui-Mhamdi
}

Volume 15, numéro 1, 2002

URI : https://id.erudit.org/iderudit/705444ar

DOI : https://doi.org/10.7202/705444ar

Aller au sommaire du numéro

\section{Éditeur(s)}

Université du Québec - INRS-Eau, Terre et Environnement (INRS-ETE)

\section{ISSN}

0992-7158 (imprimé)

1718-8598 (numérique)

\section{Découvrir la revue}

Citer cet article

Ibn Majdoub Hassani, L., Khodari, M. \& Alaoui-Mhamdi, M. (2002). Étude du régime alimentaire de deux souches de truite arc-en-ciel marocaine et bulgare dans deux plans d'eau du Moyen-Atlas Amghass I et Amghass II. Revue des sciences de l'eau / Journal of Water Science, 15(1), 165-176.

https://doi.org/10.7202/705444ar

\section{Résumé de l'article}

Le présent travail porte sur l'étude du régime alimentaire de deux souches de truites arc-en-ciel : la souche marocaine issue de croisements de truites arc-en-ciel en provenance d'Europe et d'Amérique du nord et la souche bulgare introduite au Maroc en 1987. Les milieux d'étude sont deux plans d'eau du Moyen-Atlas marocain (Amghass I et Amghass II) présentant des eaux de bonne qualité physique et chimique et une diversité biologique (invertébrés) assez importante. Afin de déterminer les préférences alimentaires de ces deux souches nous avons évalué : le spectre alimentaire, déterminé par les abondances relatives, les coefficients d'occurrence des proies ingérées, l'indice alimentaire de Lauzanne pour les différentes catégories de proies et l'intensité de prédation des truites.

Les résultats ont montré une similitude au niveau des régimes alimentaires des deux souches caractérisés par une nette préférence pour les larves et nymphes d'Insectes considérées comme proies essentielles. 


\section{Étude du régime alimentaire de deux souches de truite arc-en-ciel marocaine et bulgare dans deux plans d'eau du Moyen-Atlas Amghass I et Amghass II}

\section{Dietary regime of moroccan and bulgarian rainbow trout in two locations of the Moyen-Atlas (Amghass I and Amghass II) in Morocco}

\section{IBN MAJDOUB HASSANI ${ }^{*}$, M. KHODARI ${ }^{2}$, M. ALAOUIMHAMDI $^{1}$}

\section{SUMMARY}

This current work is about the study of the dietary regime of two species of rainbow trout. The Moroccan kind is a cross breed of the European and North American Rainbow trout and the Bulgarian trout introduced into Morocco since 1987.

The environments of study are two stretches of water in the «Moyen-Atlas» (Amghass I and Amghass II), which present water of good physical and chemical quality and are of quite important biological diversity.

To determine the dietary preferences of the two species, we evaluated several parameters: the dietary spectrum which is determined by the relative abundance and the coefficient of occurence of ingested prey, the index alimentary of Lauzanne for the different categories of prey and how predatory the trout are.

The results have shown a similarity in the level of dietary regimes of the two populations outlined by a clear preference for larvae and nymph insects which are considered to be essential prey.

Key-words: moroccan rainbow trout, bulgarian rainbow trout, dietary regime, stretch of water, insects.

1. Faculté des sciences Dhar El Mehraz, Laboratoire d'hydrobiologie et écologie générale, BP 1796, Fès Atlas, Maroc.

2. Centre national d'hydrobiologie et de pisciculture d'Azrou, BP 24, Azrou, Maroc.

* Correspondance. E-mail : leila.ibn-majdoub@caramail.com Les commentaires seront reçus jusqu'au 31 mars 2003. 


\section{RÉSUMÉ}

Le présent travail porte sur l'étude du régime alimentaire de deux souches de truites arc-en-ciel : la souche marocaine issue de croisements de truites arcen-ciel en provenance d'Europe et d'Amérique du nord et la souche bulgare introduite au Maroc en 1987. Les milieux d'étude sont deux plans d'eau du Moyen-Atlas marocain (Amghass I et Amghass II) présentant des eaux de bonne qualité physique et chimique et une diversité biologique (invertébrés) assez importante. Afin de déterminer les préférences alimentaires de ces deux souches nous avons évalué : le spectre alimentaire, déterminé par les abondances relatives, les coefficients d'occurrence des proies ingérées, l'indice alimentaire de Lauzanne pour les différentes catégories de proies et l'intensité de prédation des truites.

Les résultats ont montré une similitude au niveau des régimes alimentaires des deux souches caractérisés par une nette préférence pour les larves et nymphes d'Insectes considérées comme proies essentielles.

Mots clés : régime alimentaire, truite arc-en-ciel marocaine, truite arc-en-ciel bulgare, plans d'eau, insectes.

\section{1 - INTRODUCTION}

La truite arc-en-ciel fut introduite au Maroc dès 1925 en provenance d'Europe et d'Amérique du Nord (Mouslih, 1996). Les croisements des espèces allochtones (Oncorhychus mykiss) de différentes provenances avec d'autres espèces voisines (Salmo kamlopps et Salmo clarkil) ont donné naissance à l'actuelle souche de truite arc-en-ciel marocaine qui a montré une bonne adaptation aux eaux marocaines surtout dans la région du Moyen-Atlas (MOUSLIH, 1987).

La truite arc-en-ciel bulgare a été introduite au Maroc en 1987 en vue d'un éventuel croisement avec l'espèce marocaine pour éviter tous problèmes de consanguinité.

Ce croisement n'a pas eu lieu et le Maroc possède toujours les deux souches de truite arc-en-ciel marocaine et bulgare qui sont déversées surtout dans les plans d'eau à permis spécial gérés en parcours de pêche sportive (MOUSLIH, 1981).

Poisson de pêche sportive de grande renommée, sans oublier son importance dans le circuit touristique au Maroc, la truite arc-en-ciel demeure très peu étudiée au Maroc.

Notre travail consiste à étudier, de manière qualitative et semi-quantitative, le régime alimentaire naturel de la truite arc-en-ciel marocaine au niveau du plan d'eau d'Amghass I et de la truite arc-en-ciel bulgare au niveau d'Amghass II pendant la période allant de novembre 1998 à juillet 1999 pour la première et de juin 1998 à avril 1999 pour la deuxième, à raison d'un prélèvement par mois. 


\section{2 - MATÉRIEL ET MÉTHODES}

\subsection{Description des stations d'étude}

Les prélèvements mensuels ont eu lieu dans deux plans d'eau du MoyenAtlas marocain : Amghass I et Amghass II (figure 1), situés à 900-1 000 m d'altitude dans le causse atlasique de l'ouest et assis sur un terrain quaternaire formé par les coulées basaltiques et des dépôts essentiellement argileux issus de la décomposition sur place des calcaires et des dolomies ou de ruissellement périphérique (MOUSLIH, 1981).

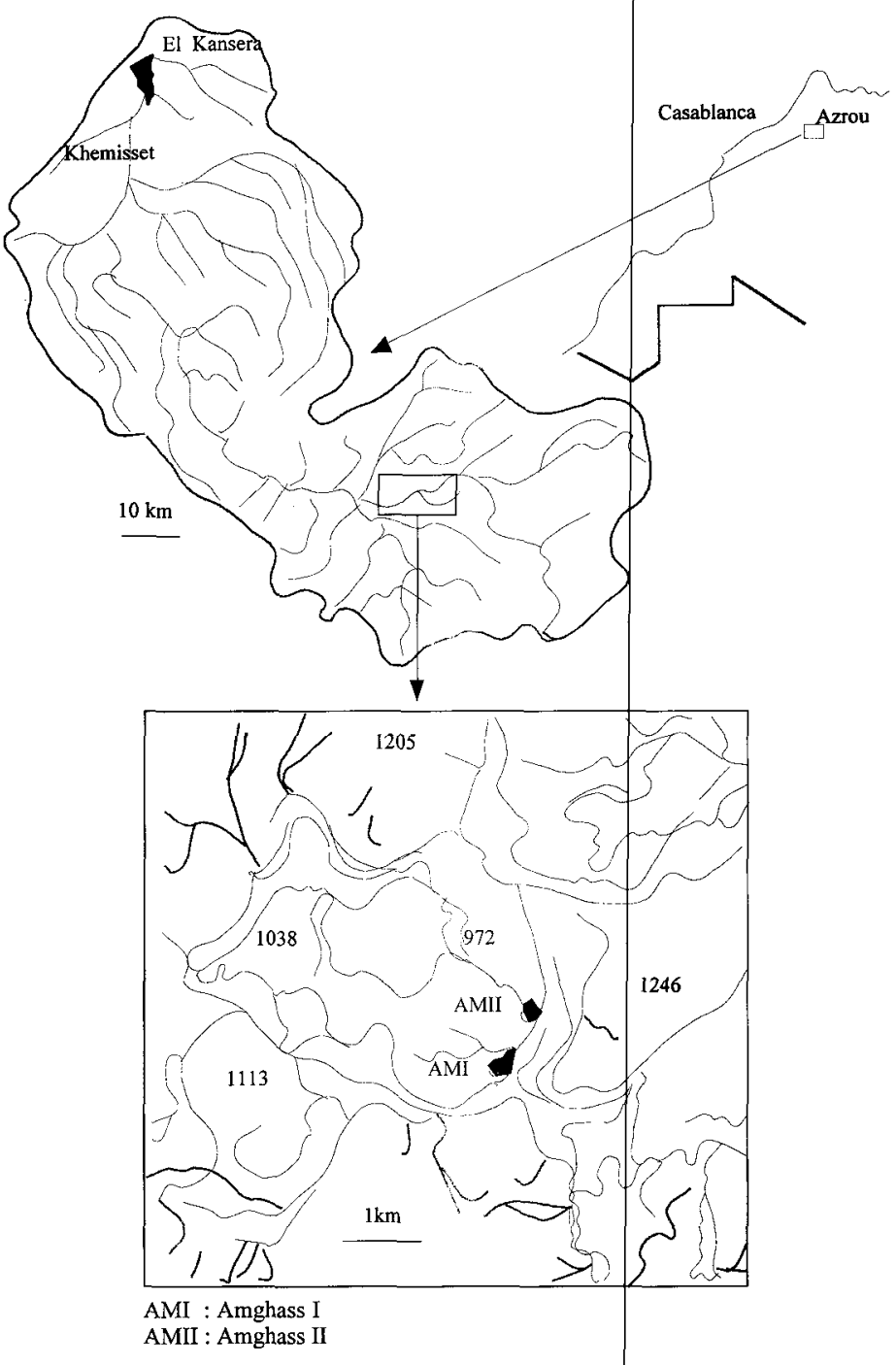

Figure 1 Localisation des sites d'études, Amghass I et Amghass II. Location of study site, Amghass I and Amghass II. 
La qualité physique et chimique des eaux (tableau 1) est très favorable pour les Salmonidés, sauf pour les chlorures qui montrent des teneurs sans doute trop élevées pour la truite.

La végétation aquatique des deux milieux est très dense et composée essentiellement de Phragmites sp., de lentilles d'eau, de Chara sp., de Zygnema $\mathrm{sp}$., de Spirogyra sp., de Myriophyllium sp. et de Ceratophyllum sp.

Tableau 1 Caractéristiques physicochimiques des plans d'eau d'Amghass I et Amghass II.

Table $1 \quad$ Physical and chemical caracteristics of water in Amghass I and Amghass II.

\begin{tabular}{|c|c|c|}
\hline Paramètre & Amghass I & Amghass II \\
\hline Oxygène dissous $(\mathrm{mg} / \mathrm{l})$ & 11,3 & 10,8 \\
$\mathrm{pH}$ & 7,8 & 7,5 \\
Conductivité $(\mu \mathrm{s} / \mathrm{cm})$ & 636 & 724 \\
Calcium $(\mathrm{mg} / \mathrm{l})$ & 82,4 & 129 \\
Magnésium $(\mathrm{mg} / \mathrm{l})$ & 22,56 & 29,5 \\
Chlorures $(\mathrm{mg} / \mathrm{l})$ & 10 & 17,6 \\
TAC $(\mathrm{mg} / \mathrm{l})$ & 392 & 444 \\
Nitrates $(\mathrm{mg} / \mathrm{l})$ & 7,27 & 5,03 \\
Orthophosphates $(\mathrm{mg} / \mathrm{l})$ & 0,01 & 0,13 \\
\hline
\end{tabular}

L'inventaire qualitatif et quantitatif des macro-invertébrés (tableau 2) montre une similitude au niveau des taxons présents dans les deux milieux, dominés essentiellement par les Nématodes, les Gastéropodes et les Achètes.

Les deux plans d'eau sont empoissonnés régulièrement par des truites de même âge (entre 6 et 9 mois) reproduites artificiellement au niveau de la station de pisciculture de Ras El Ma (à proximité de la ville d'Azrou). Huit mois plus tard, ils sont ouverts à la pêche durant deux à trois mois, puis vidangés et laissés à sec pendant une certaine période avant d'être remis en eau (SABATIÉ et al., 1984).

\subsection{Méthode de pêche et collecte des contenus stomacaux}

Comme il s'agit de plans d'eau destinés à la pêche sportive, la pêche électrique ne nous a pas été autorisée. Nous avons eu recours à la pêche à la ligne pour la capture des poissons (20 à 30 par mois).

Une fois capturées, les truites sont disséquées sur place et les estomacs mis individuellement dans des flacons avec du formol à 4 \% (NEUVEU, 1978).

$\mathrm{Au}$ laboratoire, les tubes digestifs sont vidés sous une loupe binoculaire, les proies sont isolées puis déterminées et comptées.

Afin d'évaluer le régime alimentaire de la truite arc-en-ciel (en nous basant sur le contenu stomacal moyen de l'ensemble des truites capturées pendanta la période d'étude), nous avons appliqué les techniques proposées par HYNES (1950, in SABATIÉ et al. 1984) BENABID (1990) et LAUZANNE (1975). 
Tableau 2 Inventaire faunistique des plans d'eau d'Amghass I et Amghass II.

Table 2 Fauna list of Amghass I and Amghass II.

\begin{tabular}{|c|c|c|}
\hline Taxons & Amghass I & Amghass II \\
\hline Dugesia & $\mathrm{x}$ & $\mathrm{x}$ \\
Mermithoidea & $\mathrm{x}$ & $\mathrm{x}$ \\
Naididae & $\mathrm{x}$ & $\mathrm{x}$ \\
Stylaria & $\mathrm{x}$ & $\mathrm{x}$ \\
Tubificidae & $\mathrm{x}$ & $\mathrm{x}$ \\
Helobdella & $\mathrm{x}$ & $\mathrm{x}$ \\
Erpobdellidae & $\mathrm{x}$ & $\mathrm{x}$ \\
Planorbidae & $\mathrm{x}$ & $\mathrm{x}$ \\
Physidae & $\mathrm{x}$ & $\mathrm{x}$ \\
Lymnaeidae & $\mathrm{x}$ & $\mathrm{x}$ \\
Bivalve & $\mathrm{x}$ & $\mathrm{x}$ \\
Gammaridae & $\mathrm{x}$ & $\mathrm{x}$ \\
Atyidae & $\mathrm{x}$ & $\mathrm{x}$ \\
Collemboles & $\mathrm{x}$ & $\mathrm{x}$ \\
Trichoptere & $\mathrm{x}$ & $\mathrm{x}$ \\
Éphéméroptère & $\mathrm{x}$ & $\mathrm{x}$ \\
Baetidae & $\mathrm{x}$ & $\mathrm{x}$ \\
Lestidae & $\mathrm{x}$ & $\mathrm{x}$ \\
Platycnemidae & $\mathrm{x}$ & $\mathrm{x}$ \\
Coenagrionidae & $\mathrm{x}$ & $\mathrm{x}$ \\
Mesovelidae & $\mathrm{x}$ & $\mathrm{x}$ \\
Corixidae & $\mathrm{x}$ & $\mathrm{x}$ \\
Encoilidae & $\mathrm{x}$ & $\mathrm{x}$ \\
Tipulidae & $\mathrm{x}$ & $\mathrm{x}$ \\
Culicidae & $\mathrm{x}$ & $\mathrm{x}$ \\
Ceratopogonidae & $\mathrm{x}$ & $\mathrm{x}$ \\
Tanypodinae & $\mathrm{x}$ & $\mathrm{x}$ \\
Diamasinae & $\mathrm{x}$ & $\mathrm{x}$ \\
Orthocladinae & $\mathrm{x}$ & $\mathrm{x}$ \\
Syrphidae & $\mathrm{x}$ & $\mathrm{x}$ \\
Brachycère & $\mathrm{x}$ & $\mathrm{x}$ \\
Ephydridae & $\mathrm{x}$ & $\mathrm{x}$ \\
Alevins de poissons & Amphibiens & $\mathrm{x}$ \\
Hydracariens & & \\
\hline & & $\mathrm{x}$ \\
\hline
\end{tabular}

La croix désigne la présence des taxons.

Quatre indices ont été retenus.

\subsubsection{Taux de vacuité (TV)}

II renseigne sur le rythme alimentaire des poissons, exprimé par le pourcentage d'estomacs vides (EV) par rapport aux estomacs examinés (EE).

$$
T V=(E V / E E) 100
$$




\subsubsection{Spectre alimentaire}

II permet, en fonction de l'abondance relative de chaque catégorie de proie et de son coefficient d'occurrence dans les contenus stomacaux de classer les proies en composantes permanentes ou occasionnelles.

Le Coefficient d'occurrence (CO) est le rapport, exprimé en pourcentage, entre le nombre d'estomacs contenant une catégorie de proie (EP) et le nombre d'estomacs examinés (EE).

$$
\mathrm{CO}=(\mathrm{EP} / \mathrm{EE}) 100
$$

\subsubsection{Intensité de prédation}

C'est le rapport entre le nombre total de chaque catégorie de proie $(\mathrm{Np})$ et le nombre total des estomacs examinés.

$$
\mathrm{IP}=\mathrm{Np} / \mathrm{EE}
$$

\subsubsection{Indice alimentaire (IA)}

Pour mieux cerner l'importance d'une proie dans un contenu stomacal d'un poisson, LAUZANNE (1975) a combiné l'indice volumétrique et la fréquence d'existence (occurrence) de la proie pour ainsi donner l'indice alimentaire :

$$
\mathrm{IA}=(\mathrm{CO})(\mathrm{IV}) / 100
$$

L'indice volumétrique représente le rapport en pourcentage entre le volume de chaque catégorie de proie (Vmoy) et le volume total de toutes les catégories de proie (Vtot).

$$
\text { IV }=(\text { Vmoy } N \text { tot }) 100
$$

Le volume moyen de chaque catégorie de proie est calculé à partir du volume total et du nombre total de ses individus.

Pour cet indice la composante insectes regroupe les insectes considérés comme proies permanentes ou occasionnelles. Les autres proies occasionnelles ayant été exclues de cette étude.

\section{3 - RÉSULTATS ET DISCUSSION}

\subsection{Taux de vacuité}

Le taux de vacuité des contenus stomacaux des deux populations reste nul durant toute la période d'étude. Ceci peut s'expliquer par l'agilité des jeunes truites (âge $1+$ ) qui facilite la capture des proies d'autant plus que les deux plans d'eau étudiés ont présenté durant toute l'année une alimentation suffisante.

Par ailleurs, l'impossibilité pour les deux souches de se reproduire naturellement dans les milieux étudiés et donc l'absence de période de reproduction fait que le rythme alimentaire des poissons reste assez régulier durant l'année. 


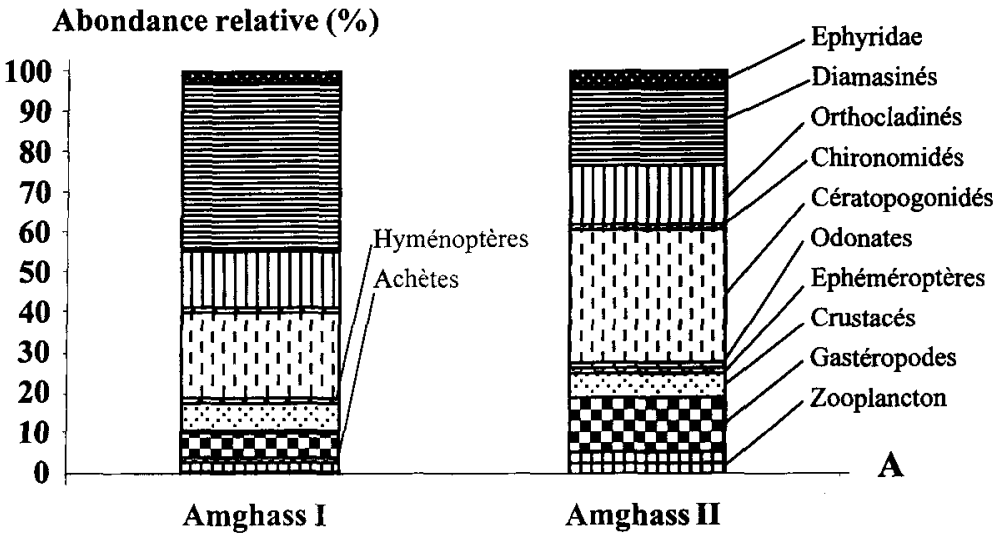

\section{Coefficient d'occurrence}

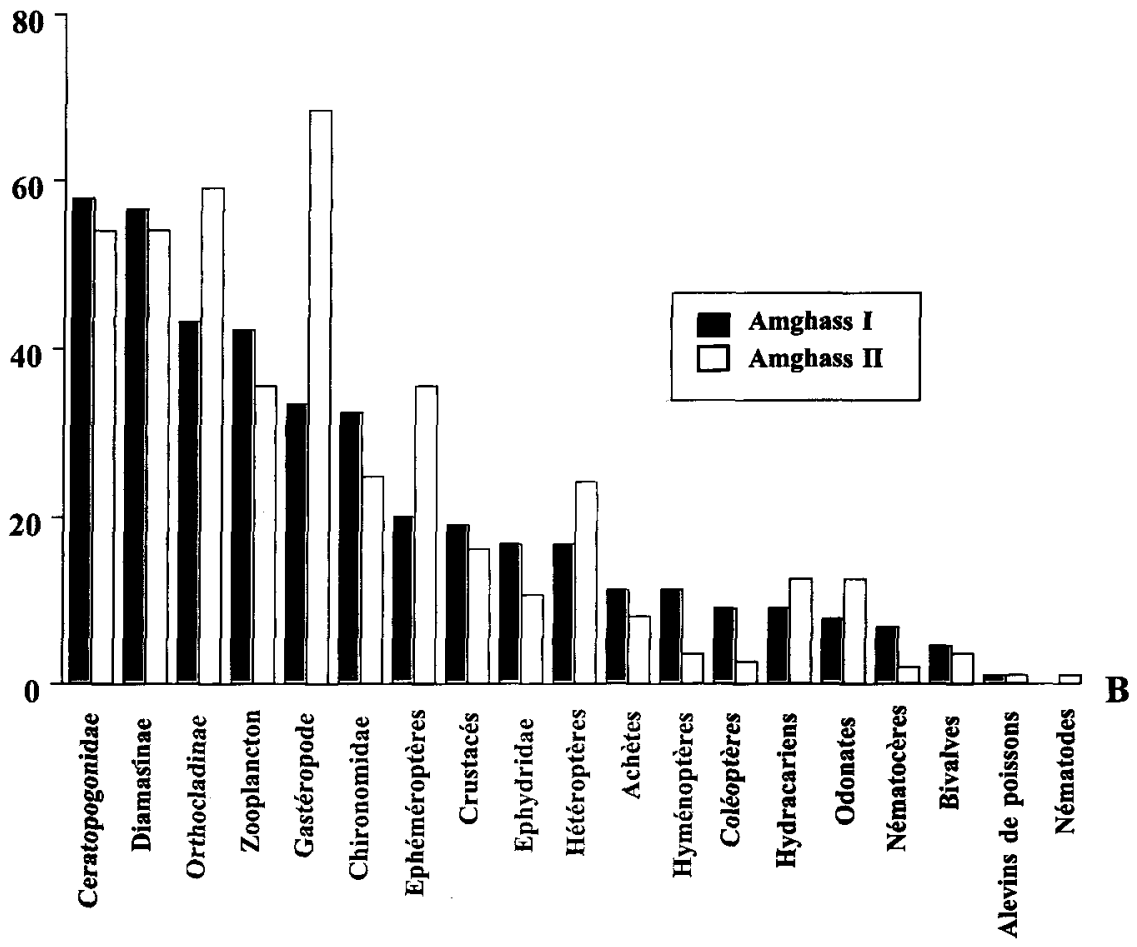

Figure 2A Abondances relatives des proies ingérées par la truite arc-en-ciel marocaine à Amghass I et par la truite arc-en-ciel bulgare à Amghass II.

Relative abundance of preys ingested by moroccan raibow trout in Amghass / and by bulgarian raibow trout in Amghass II.

Figure 2B Coefficient d'occurrence des proies ingérées par les truites arc-enciel marocaine et bulgare au niveau d'Amghass I et Amghass II.

Occurrence Coefficient of preys ingested by moroccan and bulgarian raibow trout in Amghass / and Amghass II. 


\subsection{Spectre alimentaire (figure 2)}

Par leurs abondances (valeurs moyennes sur l'ensemble de la période de prélèvement) relatives (Ar), les larves et les nymphes de Diamasinae ( $\mathrm{Ar}=41,15$ à Amghass I et 18,88 à Amghass II), de Ceratopogonidae ( $\mathrm{Ar}=20,1$ à Amghass I et 34,36 à Amghass II) et d'Orthocladinae ( $\mathrm{Ar}=13,1$ à Amghass I et 14,56 à Amghass II) sont les composantes essentielles du régime alimentaire de la truite dans les deux plans d'eau. Viennent ensuite les Gastéropodes (Physidae, Lymnaedae et Planorbidae) ( $\mathrm{Ar}=6,4$ à Amghass I et 12,35 à Amghass II), les Crustacés (Crevette d'eau douce et Gammares) (Ar $=6,47$ à Amghass I et 6,27 à Amghass II), le zooplancton (surtout les Cladocères, les Copépodes et les Ostracodes) (Ar = 3,62 à Amghass l et 5,51 à Amghass II), et quelques groupes de Chironomidae (Tanytarsini et Chironomini).

Des différences sont observées entre les deux souches au niveau de leurs préférences pour certains groupes. C'est ainsi que les Diamasinés sont plus consommés à Amghass I qu'à Amghass II, alors qu'on observe un phénomène inverse pour les Cératopogonidés, les Gastéropodes et le Zooplanctoon.

Les composantes occasionnelles sont composées d'adultes d'Hétéroptères, d'Hyménoptères et de Coléoptères, de larves d'Ephydridae, d'Odonates, de Nématocères et d'Achètes, d'Hydracariens, de Némathelminthes et de Bivalves. Les Némathelminthes ne figurant pas parmi les proies ingérées par la truite arc-en-ciel marocaine.

Par ailleurs, la présence en agrégats des larves de certaines espèces d'Insectes augmente fortement leur consommation par les truites (IVLEV, 1961). En effet, nous avons trouvé dans les contenus stomacaux de certains individus, plus d'un millier de larves de la même espèce.

L'examen des coefficients d'occurrence, bien qu'ils ne donnent pas d'indication sur l'importance quantitative des différentes proies consommées, permet de fournir une idée sur les préférences alimentaires des poissons. C'est ainsi que les Diptères Cératopogonidés (avec des coefficient d'occurrence de $57,77 \%$ à Amghass l et $53,98 \%$ à Amghass II), Diamasinés ( $\mathrm{CO}=56,66 \%$ à Amghass I et 53,98 \% à Amghass II) et Orthocladinés (CO $=43,33 \%$ à Amghass I et $59,29 \%$ à Amghass II) occupent la première place, suivis par le zooplancton, les Gastéropodes, les Chironomidés, les Éphéméroptères, les Crustacés, etc. Les Nématodes et les alevins de poissons occupent la dernière place.

Contrairement au comportement fouisseur des Cyprinidés, la truite s'intéresse très peu aux proies vivant sur le fond et dans la vase (HUET, 1970), ce qui expliquerait l'absence des Nématodes dans son régime alimentaire. Ces derniers, d'après les témoignages des pêcheurs de la région d'Amghass, semblent pourtant très bien appréciés par la truite, utilisés comme appât dans la pêche ils donnent de très bons résultats.

\subsection{Intensité de prédation (figure 3)}

Les valeurs calculées pour les deux souches de truite arc-en-ciel (valeurs moyennes sur l'ensemble de la période de prélèvement) confirment leurs préférences alimentaires constituées essentiellement d'Insectes. Ceci est probablement dû au jeune âge des individus capturés. En effet, NEVEU (1978) avait 
Indice de prédation

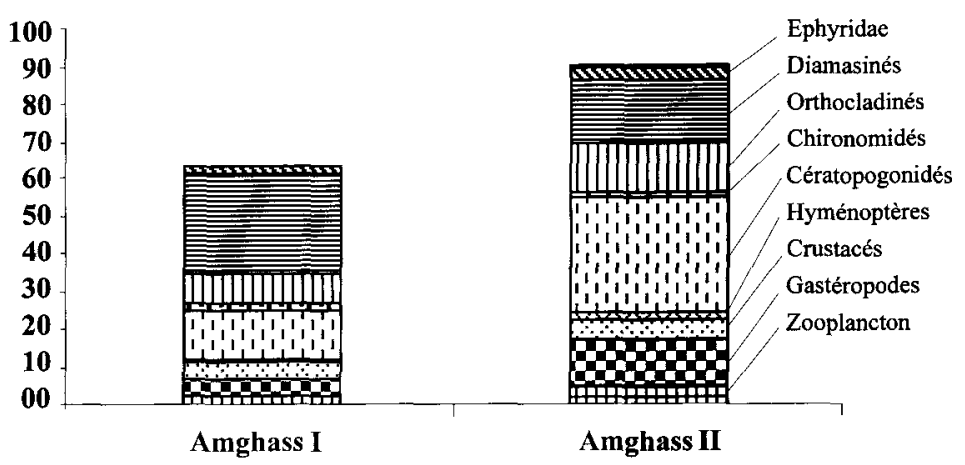

Figure 3 Intensité de prédation des truites arc-en-ciel marocaine et bulgare pour chaque catégorie de proie au niveau d'Amghass I et Amghass II.

Predatory intensity of moroccan and bulgarian raibow trout for each category of prey in Amghass I and Amghass II.

rapporté que même les poissons ichtyophages pourraient passer par une écophase où leur alimentation est constituée essentiellement par des invertébrés d'origine endogène ou exogène.

Malgré que la prédation soit plus élevée chez les truites bulgares par rapport aux truites marocaines, nous ne pouvons effectuer une comparaison " objective " entre les deux souches vu leurs différences d'âge et le décalage entre les deux périodes d'étude.

\subsection{Indice alimentaire}

Les tendances alimentaires des deux souches, selon la classification de LAUZANNE (1976), sont composées essentiellement d'Insectes (avec une nette dominance des Diptères) (IA $=38,77$ pour Amghass I contre 62,33 pour Amghass II) (tableau 3). Le zooplancton est représenté en tant que proie secondaire avec un indice alimentaire très faible.

Pour les deux souches, les Crustacés sont classés comme proies secondaires. Les Gastéropodes, classés comme proies secondaires pour la souche marocaine, sont considérés comme proies importantes pour la souche bulgare.

À travers nos résultats, la truite arc-en-ciel, poisson de physionomie plutôt carnassière, montre une tendance alimentaire nettement insectivore.

Des observations analogues ont été faites par JOHNSON (1981) sur Salmo trutta dans des affluents de la rivière Salmon aux États Unis et par JOHNSON et RINGLER (1980) sur Salmo gairdnerii dans un cours d'eau tributaire du lac Ontario. Le poisson se nourrissait principalement d'invertébrés benthiques. Dans le lac Belmore en Nouvelle Zélande, Salmo trutta et Salmo gairdnerii, se nourrissait principalement de Mollusques de petite taille (Potamopurgus antipodarum, Physa sp. et Gyraulus corinna) (MCCARTER, (1986).

En revanche, CHAMPAGNEULL et al. (1991), avaient rapporté que la truite de Lac (Salmo trutta), en particulier celle du lac Léman (pour des individus de 
Tableau 3 Indice alimentaire de LAUZANNE.

Table 3 Alimentary index of LAUZANNE.

\begin{tabular}{|c|c|c|c|c|c|c|}
\hline \multicolumn{7}{|c|}{ Truite arc-en-ciel marocaine d'Amphass I } \\
\hline Taxons & $\begin{array}{c}\text { Nombre } \\
\text { de proies }\end{array}$ & $\begin{array}{c}\text { Volume } \\
\text { moyen (ml) }\end{array}$ & $\begin{array}{c}\text { Volume } \\
\text { total (ml) }\end{array}$ & $\begin{array}{c}\text { Indice } \\
\text { volumétrique }\end{array}$ & Occurrence & $\begin{array}{c}\text { Indice } \\
\text { alimentaire }\end{array}$ \\
\hline Zooplancton & 190 & 0,0002 & 0,038 & 0,09 & 42,22 & 0,03 \\
Crustacés & 732 & 0,022 & 16,1 & 42,17 & 18,88 & 7,96 \\
Gastéropodes & 442 & 0,014 & 6,18 & 16,19 & 33,33 & 5,39 \\
Insectes & 1983 & 0,008 & 15,86 & 41,55 & 93,33 & 38,77 \\
\hline
\end{tabular}

D'après l'indice alimentaire de LAUZANNE:

- les Insectes sont des proies essentielles:

- le zooplancton, les Crustacés et les Gastéropodes sont des proies secondaires.

\begin{tabular}{|c|c|c|c|c|c|c|}
\hline \multicolumn{7}{|c|}{ Truite arc-en-ciel bulgare d'Amphass II } \\
\hline Taxons & $\begin{array}{c}\text { Nombre } \\
\text { de proies }\end{array}$ & $\begin{array}{c}\text { Volume } \\
\text { moyen (ml) }\end{array}$ & $\begin{array}{c}\text { Volume } \\
\text { total (ml) }\end{array}$ & $\begin{array}{c}\text { Indice } \\
\text { volumétrique }\end{array}$ & Occurrence & $\begin{array}{c}\text { Indice } \\
\text { alimentaire }\end{array}$ \\
\hline Zooplancton & 551 & 0,0002 & 0,11 & 0,12 & 35,39 & 0,04 \\
Crustacés & 627 & 0,022 & 13,79 & 15,1 & 15,92 & 2,4 \\
Gastéropodes & 1235 & 0,014 & 17,29 & 18,94 & 68,14 & 12,9 \\
Insectes & 7510 & 0,008 & 60,08 & 65,82 & 94,69 & 62,32 \\
\hline
\end{tabular}

D'après l'indice alimentaire de LAUZANNE :

- les Insectes sont des proies essentielles :

- les Gastéropodes sont des proies importantes;

- le zooplancton et les Crustacés sont des proies secondaires.

tailles allant de 35 à $81 \mathrm{~cm}$ ), montrait une très forte tendance à l'ichtyophagie au printemps, en été et en automne; en hiver le régime alimentaire était ichtyozooplanctonophages. Cette tendance à l'ichtyophagie de Salmo trutta a également été observée par plusieurs auteurs dans différents lacs (WOJNO, 1961 ; HOLCIK et BASTL, 1970 ; ANTONIZZA et PEROLI, 1983 ; AASS, 1984 ; BRANDT, 1986).

Nos résultats restent cependant difficilement discutables, devant le manque d'études au Maroc sur la truite arc-en-ciel.

\section{4 - CONCLUSION}

Notre travail a montré que le régime alimentaire des deux souches de truites étudiées est essentiellement insectivore avec parfois la présence d'autres composantes tels les Crustacés, les Gastéropodes, le zooplancton, les Achètes et les Hydracariens. 
Certes, la diversité de ce régime résulte sans aucun doute de la richesse du milieu mais sans toutefois refléter exactement la faune existante dans les deux milieux.

D'ailleurs, malgré la forte abondance des Nématodes, des Oligochètes et des Achètes dans les deux plans d'eau, les truites ne semblent pas les consommer, exerçant ainsi une sélection certaine sur les ressources trophiques du milieu.

La tendance ichtyophagique de la truite en tant que poisson carnassier n'a pas été clairement observée dans cette étude, probablement à cause du jeune âge des truites étudiées ou à l'accès difficile aux alevins de poissons qui s'abritent dans les berges envahies de végétation.

Il apparaît ainsi que pour mieux cerner l'alimentation naturelle des truites d'Amghass I et d'Amghass II, une étude complémentaire de l'écologie des proies s'avère nécessaire. Aussi l'étude du régime et des rythmes alimentaires journaliers de truites plus âgées pourrait expliquer les phénomènes observés.

Devant la contrainte de ne pouvoir pratiquer des prélèvements qu'en phase d'exploitation des plans d'eau et de ne pouvoir prendre qu'un nombre limité de truites, d'autres méthodes d'analyses stomacales, sans sacrifice de l'animal s'avèrent très utiles.

\section{RÉFÉRENCES BIBLIOGRAPHIQUES}

AASS P., 1984. Brown trout stocking in Norway. EIFAC symposium on stock enhacement on the management of freshwater fisheries. EIFAC Tech. Pap./Doc. Tech. CECPI, 42, Supp. 1, 123-138.

ANTONIAZZA Y,, PEDROLI J.C., 1983. Contribution à l'étude de la biologie et de la pêche de la truite de lac (Salmo trutta) dans le lac de Neuchâtel. Rapp. Comm., Pêche lac Neuchâtel, 48 p.

BRANDT S.B., 1986. Food of trout and salmon in Lake Ontario. J. Great Lakes Res, , 12, 200-205.

BENABID M., 1990. Bioécologie de deux espèces du barbeau Barbus barbus callensis (Gthr.) et Barbus labeobarbus frifschii (Val) d'un cours d'eau du Haut-Atlas du Maroc. Thèse de $3^{\ominus}$ cycle, Fac. Sci. Marrakech, 170 p.

HUET M., 1970. Traité de pisciculture, $4^{e}$ édition, DE WYNAERT, Bruxelles.

HOLCIK J., BASTL I., 1970. Notes on the biology and origin of the trout (Salmo trutta $\mathrm{m}$. lacustris) in the Orava valley reservoir (Northern Slavakia). Zool. List., 19, 71-85.

HYSLOP E.J., 1980. Stomach contents analysis. A review of methods and their application. J. Fish Biol., 17, 411-429.

IVLEV V.S., 1961. Experimental ecology of the feeding of fishes. Transl. D. Scott. Yale Univ. Press, New Haven, Conn, 302 p.

JOHNSON J.H., 1981. Food interrelationships of coexisting brook trout, brown trout and yearling raibow trout in tributaries oh the Salmon river, New York. New York Fish and Game Journal, 28, 88-99.

JOHNSON J.H., RINGLER N.H., 1980. Diests of juvenile coho salmon (Oncorhynchus kisutch) and steelhed trout (Salmo gairdnerii) relative to prey avaibility. Can. J. Zool., 58, 553-558.

LAUZANNE L., 1975. Régime alimentaire d'Hydrocyon forskalii (pisces Characidae) dans le lac Tchad et ses tributaires. Cah. ORSTOM, Série Hydrobiol., Vol IX (2), 105-121.

LAUZANNE L., 1976. Régime alimentaire et relations trophiques des poissons du lac 
Tchad. Cah. ORSTOM, série hydrobiol., Vol X (4), 267-310.

MC CARTER N.H., 1986. Food and energy in the diet of brown and rainbow trout from lake benmore, New Zeland. New Zeland Journal of Marine and Freswhater Research, 20, 551-559.

MOUSLIH M., 1987. Introduction de poissons et d'ecrevisses au Maroc. Rev. Hydrobiol. trop., 20 (1), 65-72.

MOUSLIH M., 1981. Contribution à l'étude de la biologie de la truite arc-en-ciel: Salmo gardneiri, Richardson, dans un plan d'eau artificiel du Moyen-Atlas. Mem. Ing. IAV Hassan II, Rabat.

MOUSLIH M., 1996. Contribution à l'amélioration des techniques de salmoniculture de repeuplement en vue de restaurer la truite fario : Salmo trutta macrostigma,
Duméril, 1858 dans les rivières des montagnes marocaines. Mémoire d'ingénieur en chef. Ministère de l'Agriculture et de la Mise en Valeur Agricole, Rabat, $120 \mathrm{p}$.

NEVEU A., 1978. Les problèmes posés par l'étude de l'alimentation naturelle des populations sauvages de poissons. Bull. Centre Et., Rech. Sci. Biarritz Fra., 3, 501-512.

SABATIE M.R., HERMAK A., MOUSLIH M., 1984. Études hydrobiologiques d'un cours d'eau et d'un étang artificiel du Moyen-Atlas. Éléments de la démographie des populations de Salmonidés. Actes inst. Agro. Vét., 4 (1), Spécial Zoologie, 165-179.

WONJO T., 1961. The feeding of trout (Salmo trutta) from Wdzydze Lake. Rocz. Nauk. Roln., 93, 681-702. 